\title{
Morphology of Holographic Polymer Dispersed Liquid Crystal Reflection Gratings Written in Thiol-ene and Acrylate Polymer Hosts: Part II-The Effect of Diamond Knife Angle and Sectioning Direction in Ultramicrotomy
}

\author{
D.W. Tomlin, ${ }^{* *}$ L.V. Natarajan, ${ }^{*}$ V.P. Tondiglia, ${ }^{*}$ R.L. Sutherland,* and T.J. Bunning
}

Air Force Research Laboratory, Materials and Manufacturing Directorate, WPAFB, OH 45433

*Science Applications International Corporation, 4031 Col. Glenn Highway, Dayton, OH 45431

**UES, Inc, 4401 Dayton-Xenia Road, Dayton, OH 45432

In our labs, the morphology of holographic reflection gratings in polymer-dispersed liquid crystals (H-PDLC) formed in multifunctional acrylate systems have normally been investigated in detail by high-resolution low voltage scanning electron microscopy (HRLVSEM), utilizing the Hitachi S5200-UHR scanning electron microscope. The grating films formed by the acrylate system are typically glassy and are easily separated from the cell used in writing. These films can then be mounted and fractured in liquid nitrogen for cross-sectional analysis (Figure 1(a)). However, due to the elastomeric nature of the thiol-ene grating films, the same type of cross-sectional analyses became inherently more complex. The elastic nature of the thiol-ene grating was somewhat reduced by pre-staining the film with ruthenium tetroxide vapor $\left(\mathrm{RuO}_{4}\right)$, this allows the film to be "peeled" and makes it much easier to handle in general (Figure 1(b)). The films are typically soaked in methanol from 30-40 minutes, mounted in cross-section and fractured in liquid nitrogen. The sample is then coated with 10-25 $\AA$ of tungsten, using a South Bay Technology, Inc. IBS/e Sputter Coater. We have noted in our extensive HRLVSEM studies of these systems, that there can be appreciable shrinkage of the grating spacing of these systems. We believe this is due to the collapse of the grating region caused by the removal of the liquid crystal (LC) domains.

To this end, we have employed bright field transmission electron microscopy (BF TEM). The samples are prepared for ultramicrotomy, by first "peeling" the film from the glass. The sample is then trimmed and embedded in EPO FIX resin and allowed to cure, the resultant blocks are then trimmed and are ready for sectioning. Some of our preliminary work on the acrylate H-PDLCs utilizing a DiATOME $55^{\circ}$ diamond knife indicated that the grating spacing was again subject to shrinkage. Since the LC is not extracted prior to embedding, there must be another mechanism responsible for this shrinkage. It is believed that this shrinkage is actually due more to the compression of the sections caused by the use of the $55^{\circ}$ diamond knife during ultramicrotomy, than to actual shrinkage. We then set out to characterize the effects of ultramicrotomy on these systems.

Three brand new diamond knives were purchased from DiATOME U.S. The three knives chosen from the Ultra series were the $55^{\circ}, 45^{\circ}$, and $35^{\circ}$. Each grating was again prepared and embedded in EPO FIX. The acrylate and thiol-ene blocks were sectioned with each of the three knives, with the grating parallel and perpendicular to the sectioning direction. The 50-70 nm sections of the samples were obtained at room temperature utilizing a Reichert Ultracut. The sections were examined "as cut" and were also stained with ruthenium tetroxide $\left(\mathrm{RuO}_{4}\right)$ vapor, to increase contrast and to make the sample more stable in the electron beam. The samples were examined in bright field utilizing the FEI CM200 LaB 6 TEM. Figure 2(a) shows the thiol-ene grating sectioned with the DiATOME Ultra35 diamond knife, parallel to the grating direction and stained with $\mathrm{RuO}_{4}$. Figure $2(\mathrm{~b})$ is representative of the thiol-ene grating sectioned with the DiATOME Ultra55 ${ }^{\circ}$ diamond knife, 
perpendicular to the grating direction. Figure 2(c) depicts the acrylate grating sectioned with the DiATOME Ultra $45^{\circ}$ diamond knife, perpendicular to the grating direction. The arrow in each figure shows the cutting direction and Table 1 summarizes the grating spacings as measured from the thiolene system. The acrylate system shows comparable results. As expected the greatest amount of compression was found in those samples sectioned with the DiATOME $55^{\circ}$ diamond knife, perpendicular to the grating direction, while those samples sectioned parallel to the grating with the DiATOME $35^{\circ}$ diamond knife, show little to no compression. Staining with $\mathrm{RuO}_{4}$ vapor seemed to have little effect on the gratings themselves, except to enhance electron contrast.

Table 1:

\begin{tabular}{|c|c|c|c|c|c|c|}
\hline \multirow[b]{2}{*}{ Sample } & \multirow[b]{2}{*}{ Method } & \multirow[b]{2}{*}{ Cut direction } & \multicolumn{4}{|c|}{ Grating Spacing (nm) } \\
\hline & & & $\begin{array}{c}\text { LN2 } \\
\text { fracture }\end{array}$ & $\begin{array}{l}\text { DiATOME } \\
\text { Ultra35 }^{\circ}\end{array}$ & $\begin{array}{l}\text { DiATOME } \\
\text { Ultra } 45^{\circ}\end{array}$ & $\begin{array}{c}\text { DiATOME } \\
\text { Ultra55 }\end{array}$ \\
\hline Thiol-ene $(150 \mathrm{~nm})$ & BFTEM & parallel & & $153 \pm 9$ & $153 \pm 10$ & $138 \pm 10$ \\
\hline Thiol-ene & BFTEM & perpendicular & & $129 \pm 10$ & $138 \pm 9$ & $137 \pm 6$ \\
\hline Thiol-ene (stain) & BFTEM & parallel & & $151 \pm 6$ & $148 \pm 6$ & $150 \pm 7$ \\
\hline Thiol-ene (stain) & BFTEM & perpendicular & & $135 \pm 7$ & $135 \pm 3$ & $136 \pm 6$ \\
\hline Thiol-ene (stain) & HRSEM & & $136 \pm 2$ & & & \\
\hline Acrylate $(154 \mathrm{~nm})$ & HRSEM & & $110 \pm 3$ & & & \\
\hline Acrylate & BFTEM & perpendicular & & & $115 \pm 4$ & \\
\hline
\end{tabular}

Figure1:

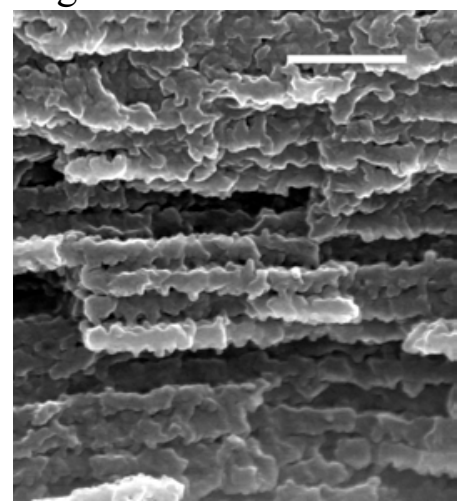

(a) scale bar $500 \mathrm{~nm}$

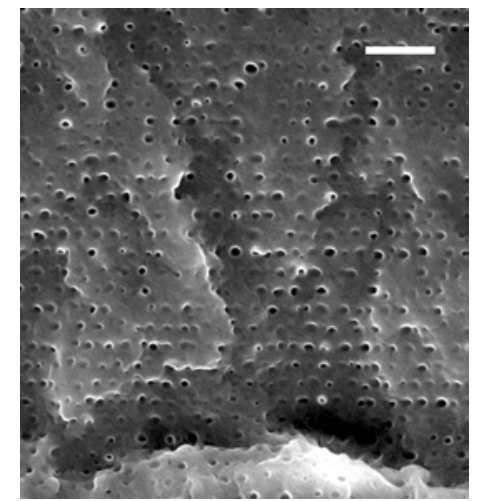

(b) scale bar $500 \mathrm{~nm}$

Figure2:

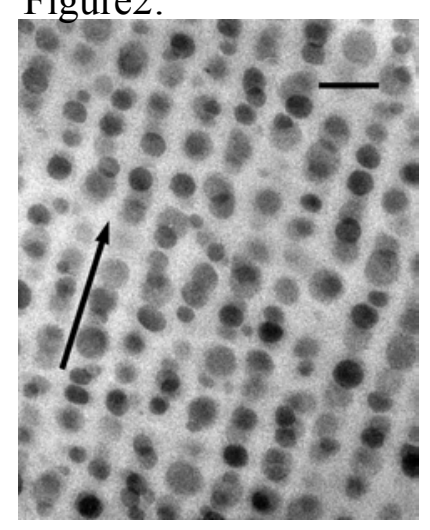

(a) scale bar $200 \mathrm{~nm}$

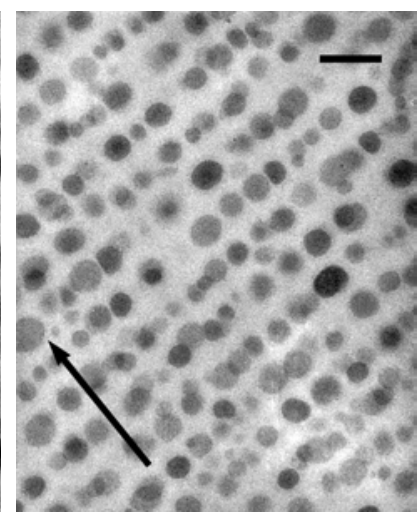

(b) scale bar $200 \mathrm{~nm}$

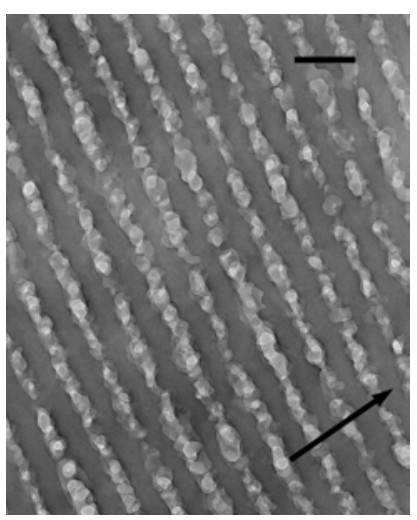

(c) scale bar $200 \mathrm{~nm}$

Arrows indicate sectioning direction. 